\title{
Focal Varieties of Curves of Genus 6 and 8
}

\author{
A. Bajravani \\ Department of Pure Mathematics, Faculty of mathematical sciences, \\ Tarbiat Modares University, Tehran, Iran \\ P. O. Box 14115-134
}

\begin{abstract}
In this paper we give a simple Torelli type theorem for curves of genus 6 and 8 by showing that these curves can be reconstructed from their Brill Noether varieties. Among other results, it is shown that the focal variety of a general, canonical and nonhyperelliptic curve of genus 6 , is a hypersurface.
\end{abstract}

AMS Classification: 14M99; 14D22; 14H20; 14H51

Keywords: Focal variety; First order foci; Moduli space; Plane quintic; Brill Noether theory.

\section{Introduction}

In a series of papers, [1, 2] and [3, C. Ciliberto and E. Sernesi showed that a canonical, nonhyperelliptic curve of genus $g$, can be reconstructed from its Brill-Noether varieties. They proved that $C_{g-1}^{1}$ parameterizes a family of linear subspaces of $\mathbf{P}^{g-1}$, which can determine the canonical curve, uniquely ( See [2] ). A canonical curve is a non-hyperelliptic curve of genus $g$ which is embedded in $\mathbf{P}^{g-1}$ by its canonical line bundle. In these papers they use the technique of first order foci. Briefly, first order foci is the locus where the rank of some special morphism drops. For a general element of the parameter space in [2, first order foci is a rational normal curve in some linear subspace of $\mathbf{P}^{g-1}$. The union of these rational curves, called focal variety, contains the canonical curve and specifically in genus 5 case, it is actually a covariant of a net of quadrics in $\mathbf{P}^{g-1}$ (See [3]). In prolongation of the works [1, 2] in [3, for a general canonical curve of genus $g=2 n+1$, they use $C_{n+2}^{1}$, where they could use second order foci, and they got a Torelli type theorem for general curves of odd genus (See [3]). In that work, they show that the focal variety of a general nonhyperelliptic and canonical curve of genus 5 is a hypersurface. We show that this

E-mail Address: bajravani@modares.ac.ir 
fact is valid also for genus 6 case. The fact that, the focal variety of a general curve of genus 6 is of expected dimension, implies that our computation of second order foci in genus 6 case is valid.

Throughout this paper, we will work over an algebraically closed field of characteristic zero. Assume that $C$ is a general canonical non-hyperelliptic curve of genus 6 (res. of genus 8 ), which is embedded by its canonical line bundle in $\mathbf{P}^{5}$ (res. in $\mathbf{P}^{7}$ ). As it is usual in Brill-Noether theory, $\mathbf{g}_{d}^{r}$ denotes a line bundle of degree $d$ with global sections of dimension at least $r+1$. We denote by $W_{d}^{r}$ the set of $\mathbf{g}_{d}^{r}$ 's, and we set $W_{d}=W_{d}^{0}$. It is known in Brill-Noether theory that $W_{d}^{r}$ has a scheme structure and for a general curve it is an irreducible nonsingular variety, unless it has dimension zero. We will denote the set of effective divisors of degree $d$ with global sections of dimension at least $r+1$ by $C_{d}^{r}$. The morphism: $\alpha_{d}: C_{d} \rightarrow W_{d}$ is the Abel- Jacobi map. Also, $\mathbf{M}_{g}$ denotes the moduli space of curves of genus $g$.

This paper consists of three sections. In the section 2 , we prove that the focal variety of a general canonical and non-hyperelliptic curve of genus 6 , is a hypersurface. Then, by describing the second order foci in this case, we will give a short proof of a Torelli type theorem for such a curve. We see that a general canonical nonhyperelliptic curve of genus 6 , is an irreducible component of the closure of the union of its second order foci. We also give examples to show that some components of the focal scheme may be of lower dimension than expected, for special curves. In section 3 , for general canonical and nonhyperelliptic curves of genus 8 , we prove that the first order foci are, in general, an irreducible cubic threefold in a linear subspace of dimension 4: in one of the possible cases, their singular locus is a rational quartic curve and the first order foci is the secant variety of the rational quartic curve. Also, using similar argument as in [2] and [3], we show that the union of a certain 1-parameter family of these rational curves, is a rational surface, which is used to prove Torelli with this approach.

\section{The genus 6 case}

\subsection{First Order Foci and Focal Variety}

Consider the scheme $W_{5}^{1}$, which is a 2-dimensional variety for a general curve of genus 6 . By generality condition the Abel-Jacobi map is a $\mathbf{P}^{1}$-bundle. So $C_{5}^{1}$ is a 3 -fold, which we denote by $S$. For a divisor $D_{s} \in S$, let $\Lambda_{s}=\overline{D_{s}}$ be the three dimensional linear space spanned by $D_{s}$. These linear subspaces fit together to make a 3 -dimensional family of 5-secant linear subspaces of $\mathbf{P}^{5}$ parameterized by $S$ :

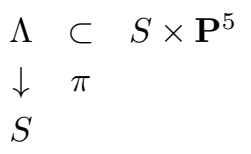


For more details about first order foci, see [2] and [3].

Theorem 1. For a general $L \in W_{5}^{1}$ and for all sufficiently general $s \in \alpha_{5}^{-1}(L)$, the focal variety $F_{s}$ at $s \in S$, is a rational normal cubic curve in $\Lambda_{s}$.

Proof. By the Brill-Noether theorem for a general line bundle $L \in W_{5}^{1}$ on a general curve, the linear series $|L|$ is base point free (See [6]). Now, the theorem follows by 2.5 of [2].

Remark 1. Note that, Theorem 1 is valid without generality assumption on the line bundle $L$, and we will come back to its proof in Theorem 7 . But for our aim in this section it is enough to take a general line bundle $L \in W_{5}^{1}$.

For a line bundle $L \in W_{5}^{1}$, the irreducible variety $Q=\cup_{s \in \alpha_{5}^{-1}(L)} \Lambda_{s}$ is a quadric hypersurface with vertex a line which we denote by $\Gamma$.

Lemma 2. For any $s \in \alpha_{5}^{-1}(L)$, the rational normal curve $F_{s}$ cuts the vertex $\Gamma$ in two points.

Proof. This has been proved originally in proposition 4.2 of 2 .

For a fixed line bundle $L \in W_{5}^{1}$, the union $\cup_{s \in \alpha_{5}^{-1}(L)} F_{s}$ is birational with $\mathbf{P}^{1} \times \mathbf{P}^{1}$ and under this birationality, the lines $\{p t\} \times \mathbf{P}^{1}$ are mapped to focal curves (see [2]). Let $F_{C}=\overline{\cup_{s \in S} F_{s}}$ be the focal variety of the canonical curve $C$. We prove that $F_{C}$ is a hypersurface.

Lemma 3. Let $\mathbf{M}_{g}$ be the moduli space of curves of genus $g$. Define a map:

$$
\begin{gathered}
\Psi: \quad \mathbf{M}_{g} \rightarrow \mathbb{Z} \\
\Psi([C])=\operatorname{dim}\left(F_{C}\right)
\end{gathered}
$$

Then $\Psi$ is a lower-semicontinuous function.

Proof. This follows from the fact that $\operatorname{dim}\left(F_{C}\right)=\operatorname{dim}\left(V\left(\chi_{C}\right)\right)-\operatorname{dim}\left(V\left(X_{C}\right)_{t}\right)$ where $V\left(\chi_{C}\right) \subset$ $S \times \mathbf{P}^{g-1}$ is the focal locus, $f_{C}: V\left(\chi_{C}\right) \rightarrow \mathbf{P}^{g-1}$ is the projection and $V\left(X_{C}\right)_{t}$ is a general fibre of $f_{C}$ for a general $t \in \mathbf{P}^{g-1}$. Now, we have a map $\phi: \cup_{C \in \mathbf{M}_{g}} V\left(\chi_{C}\right) \rightarrow \mathbf{P}^{g-1} \times \mathbf{M}_{g}$ which restricts to $f_{C}$ over $C$. By [8, page 95], we know that the dimension of the general fibre of $\phi$ is uppersemicontinuous as a function of $C$, which gives the upper-semicontinuity of the general fibre of $f_{C}$. Now it follows that $\operatorname{dim}\left(F_{C}\right)$ is lower semicontinuous as a function of $C$.

Note that using this lemma it is enough to find a special curve whose focal variety has a component which is a hypersurface. The following theorem gives an answer to this question. 
Theorem 4. Let $C$ be a smooth plane quintic. Then $F_{C}$ is a hypersurface.

Proof. For a smooth plane quintic curve $C$, a general element of $S$ is of the form:

$$
D_{s}=p_{1}+p_{2}+p_{3}+p_{4}+q
$$

where $p_{1}+\cdots+p_{5}$ is a general element of the $g_{5}^{2}$ and $q$ is a general element of $C$.

The Veronese embedding maps the line passing through $p_{1}+\cdots+p_{5}$ to a conic $\Gamma$ in $\mathbf{P}^{5}$. Also, it maps the tangent line to $C$ at $q$, to a conic $\bar{\Gamma}$ and we have $\Gamma \cap \bar{\Gamma}=\{p\}$, and the focal curve is $\Gamma \cup \overline{p, q}$. So visibly $F_{C}$ consists of two parts. One is a union of conics $\Gamma$, which is two dimensional, because all of them are contained in the Veronese surface. The other part is a union of lines, which we want to prove it is a fourfold. The above argument proves that the focal variety consists of all the "join" of the veronese surface with $\mathrm{C}$ (i.e. the closure of the union of lines spanned by a general point q of $\mathrm{C}$ and a general point of the Veronese). Now, the lines in this family through $\mathrm{q}$ form a cone over the Veronese with vertex q. So their union has dimension 3. Now, moving $q$ on $C$ leads us to a 1-dimensional family of 3-dimensional cones over the Veronese surface, with vertices moving on $C$. Therefore we will have $\operatorname{dim}\left(F_{C}\right)=4$.

Consider that in this case the canonical curve $C$ and the Veronese surface $F$ are contained in the focal variety $F_{C}$. Now by Lemma 3 and Theorem 4, we obtain the following

Theorem 5. Let $C$ be a general canonical and nonhyperelliptic curve, of genus 6 . Then its focal variety, $F_{C}$, is a hypersurface in $\mathbf{P}^{5}$.

Note that, without generality assumption on the curve $C$, Theorem 5 is not valid. In general the following examples show that some components of the focal scheme may be of lower dimension than expected.

Example 1. Let $C$ be a bielliptic curve of genus $g$. In this case $C_{g-1}^{1}$ has a component $S$, whose general member is of the form $D_{s}=P_{1}+P_{2}+Q_{1}+Q_{2}+R_{1}+\cdots+R_{g-5}$, where the lines $\overline{P_{1} P_{2}}$ and $\overline{Q_{1} Q_{2}}$ are two generators of the elliptic cone, and $R_{1}, \ldots, R_{g-5}$ are general points of $C$. In this setting we have

$$
F_{s}=\overline{P_{1} P_{2}} \cup \overline{Q_{1} Q_{2}} \cup \overline{O R_{1}} \cup \cdots \cup \overline{O R_{g-5}}
$$

For more details about constructions of focal loci in exceptional cases see 2]. Now it easy to see that the focal variety is of dimension 2 . In fact by taking $P_{1}, P_{2}, Q_{1}, Q_{2}$ fixed and by moving $R_{i}$, $(1 \leq i \leq g-5)$, on the curve we will get a cone over $C$ with vertex $O$, which is of dimension 2 .

In bielliptic case, $C_{g-1}^{1}$ has a component whose general element is the residual of the general element of $\mathrm{S}$ with respect to the canonical series. It is not known, whether or not, the focal variety over this component is a hypersurface. 
Example 2. Let $C$ be a trigonal curve of genus $g \geq 6$. In this case $C_{g-1}^{1}$ has a component $S$, whose general member has the form $D_{s}=P+Q+R+P_{1}+\cdots+P_{g-4}$, where $P+Q+R \in \mathbf{g}_{3}^{1}$ and $P_{1}+\cdots+P_{g-4}$ are general points of $C$. Let $\underline{r}$ denotes the line joining the points $P, Q$ and $R$. The irreducible scheme $S$ has dimension $g-3$. Take $S$ as the parameter space of the family of linear subspaces of $\mathbf{P}^{g-1}$ spanned by divisors $D_{s}$, in which $D_{s}$ is a general member of $S$. As in the previous example the expected dimension is $g-2$. In this setting we have

$$
F_{s}=\underline{r} \cup \overline{P_{1} Q_{1}} \cup \cdots \cup \overline{P_{g-4} Q_{g-4}} .
$$

The nature of the points $Q_{i}$ is explained completely in [2]. For our aim it is enough just to know that they are points on the line $\underline{r}$, which is the line passing through points $P, Q, R$. It is easy to see that the focal variety is of dimension at most 4 . In fact for a line $L$ generated by a general member $P+Q+R$ of $\mathbf{g}_{3}^{1}$, set $F_{L}=\bigcup_{P_{1}, \ldots, P_{g-4}} F_{P_{1}, \ldots, P_{g-4}}$, where $F_{P_{1}, \ldots, P_{g-4}}=\underline{r} \cup \overline{P_{1} Q_{1}} \cup \cdots \cup \overline{P_{g-4} Q_{g-4}}$, and $P_{1}, \ldots, P_{g-4}$ moves on the curve. Consider that $F_{L}$ is contained in the joining variety of $C$ and the line $L$, so $F_{L}$ has dimension at most 3. By moving $L$ in $\mathbf{g}_{3}^{1}$ we will get a 1-dimensional family of $F_{L}$ 's, whose union, $F_{C}$, has dimension at most 4 .

Also in this case $C_{g-1}^{1}$ has another component and again it is not known whether or not the focal variety on this component is a hypersurface.

\subsection{Second order Foci in genus 6 case}

We have seen that the family (1) defines, for $s \in S$, a closed subscheme $F_{s} \subset \Lambda_{s}$, whose points are the so called first order foci at $s$. All these subschemes fit together in a closed subscheme $\mathcal{F} \subset \Lambda$ and we obtain a morphism $\pi_{1}: \mathcal{F} \rightarrow S$ and a diagram :

$$
\begin{array}{lll}
\mathcal{F} & \subset & S \times \mathbf{P}^{5} \\
\downarrow & \pi_{1} & \\
S & &
\end{array}
$$

By Theorem 1 (or by Theorem 8), it follows that for all sufficiently general $s \in S$ the fibre $F_{s}=\pi_{1}^{-1}(s)$, is a rational normal curve in $\Lambda_{s}$. For such an $s$, we can introduce the second order foci of the family (11), defined as the first order foci of the family (2) at $s$. For more details about second order foci see [3]. We will denote by $D_{1}\left(\xi_{s}\right)$, the second order foci of the family (1) at $s$.

Lemma 6. The second order foci in the genus 6 case is a divisor of the form $2 E+F$ over the focal curve, where $E=p_{1}+\cdots+p_{5}$ and $F$ is a divisor of degree 2 . In fact $F$ is the intersection locus of the vertex $\Gamma$ with the rational normal curve $F_{s}$.

Proof. By the same computation as in [3] we have that $\operatorname{deg}\left(D_{1}\left(\xi_{s}\right)\right) \leq 12$. The points $p_{1}, \ldots, p_{5}$ belong to $D_{1}\left(\xi_{s}\right)$, because they belong to the two dimensional family of linear spaces spanned by 
the divisors which contain the point $p_{i}$ in their support. By the same reason the points $p$ and $q$, which are the intersection points of $F_{s}$ with $\Gamma$, belong to $D_{1}\left(\xi_{s}\right)$. Now, as an application of Porteous formula, it is easy to see that the points $p_{1}, \ldots, p_{5}$ appear with multiplicity at least two. So they have multiplicity two in $F_{s}$. Therefore $p$ and $q$ appear with multiplicity one and we are done.

Corollary 7. The canonical curve $k(C)$ can be constructed uniquely from the family (1). In fact it is a component of the closure of the union of its second order foci.

During preparing this paper, C. Ciliberto and E. Sernesi pointed out to me the paper [4], where similar and more general computations of second order foci have been done.

\section{The genus 8 case}

\subsection{First order foci and its singular locus}

In the genus 8 case take $S=C_{6}^{1}$, as parameter space, which is a threefold; so we will have 3-dimensional family of $\Lambda_{s}$ 's inside $\mathbf{P}^{7}$. For a general $s \in S$ the morphism:

$$
\Phi_{s}: T_{S, s} \otimes O_{\Lambda_{s}} \rightarrow N_{\Lambda_{s} / \mathbf{P}^{7}}
$$

is a morphism between vector bundles of rank 3. Consider the first order foci $F_{s}=\{x \in$ $\left.\Lambda_{s} \mid r k\left(\Phi_{s}(x)\right) \leq 2\right\}$. For a fixed line bundle $L \in W_{6}^{1}$, the rational normal scroll $H_{L}=\cup_{D_{s} \in \alpha_{6}^{-1}(L)} \Lambda_{s}$ has a line $\Gamma$ as it's vertex. The vertex $\Gamma$ is contained in $F_{s}$, because it is contained in the 1-parameter family of $\Lambda_{s}$ parameterized by $\mathbf{P}^{1} \cong \alpha_{6}^{-1}(L)$.

Theorem 8. For a sufficiently general $s \in S, \Phi_{s}$ is 1-generic. In particular $F_{s}$ is an irreducible cubic threefold.

Proof. By generality condition on the curve, it follows that $\left|L^{2}\right|$ is a $\mathbf{g}_{g+4}^{4}$ and it is not composed with an involution. Now the proof of this theorem is similar to the proof of theorem 2 of [3] to which we refer.

Now set $\Gamma_{s}=\left\{x \in \Lambda_{s} \mid r k\left(\Phi_{s}(x)\right) \leq 1\right\}$. By corollary 3.3 of [5], we have $\operatorname{codim}_{\Lambda_{s}} \Gamma_{s} \geq 3+3-3=$ 3 , or equivalently $\operatorname{dim} \Gamma_{s} \leq 4-3=1$. So there are two cases. First suppose that $\operatorname{dim} \Gamma_{s}=0$. In this case as an application of Porteous formula we find that $\operatorname{deg}\left(\Gamma_{s}\right)=6$. Set $D_{s}=p_{1}+\cdots+p_{6}$. Since by the same reasons as in lemma $6, p_{i}(1 \leq i \leq 6)$, belongs to $\Gamma_{s}$, we have that $\Gamma_{s}=\left\{p_{1}, \ldots, p_{6}\right\}$. Now suppose that $\operatorname{dim}\left(\Gamma_{s}\right)=1$. In this case by theorem 5.1 of $\left[5, \Gamma_{s}\right.$ is a rational normal curve 
and $\Phi_{s}$ is a Catalecticant matrix, so $F_{s}$ is the secant variety of $\Gamma_{s}$. In the second case consider the morphism

$$
\Psi_{s}: T_{S, s} \otimes O_{\Gamma_{s}} \rightarrow N_{\Gamma_{s} / \mathbf{P}^{7}}
$$

which is a morphism between vector bundles of ranks 3 and 6 , respectively. Consider the locus where the rank of $\Psi_{s}$ drops twice, i.e. the set $H_{s}=\left\{x \in \Gamma_{s} \mid r k\left(\Psi_{s}(x)\right) \leq 1\right\}$. By the methods of the paper [3], we find that $\operatorname{deg}\left(H_{s}\right)=8$. Also, we know that $p_{i}(1 \leq i \leq 6)$ belong to $H_{s}$. So we will have the following corollary, whose proof we will complete in the next subsection.

Corollary 9. The canonical curve $k(C)$ can be constructed uniquely from a family, analogous to the family (1). Precisely in the first case, $k(C)$ is the closure of union of the singular locus of first order focis. In the second case the canonical curve $k(C)$ is a component of the closure of the union of those loci, where $\Psi_{s}$ 's, drops rank twice.

\subsection{Focal surfaces}

For a canonical curve of genus 8 , assume that we are in the second case and for a general line bundle $L \in W_{6}^{1}$ denote by $\mathbf{U} \subset \alpha_{6}^{-1}(L)$ the open subset such that $\Gamma_{s}$ is a rational normal curve. Define:

$$
F_{L}=\overline{\cup_{s \in \mathbf{U}} \Gamma_{s}}
$$

$F_{L}$ is an irreducible surface. By generality assumption the morphism $\phi_{L^{2}}: C \rightarrow \mathbf{P}^{4}$ maps $C$ birationally onto a curve of degree 12 . By base point free pencil trick and generality condition on the curve $C$, the multiplication map

$$
\bar{\mu}_{L}: H^{0}(C, L) \otimes H^{0}(C, L) \rightarrow H^{0}\left(C, L^{2}\right)
$$

is an injective map, and so the projectivized of dual of it's image is a point $p$ in $\mathbf{P}^{4}$ which can be realized as the vertex of a quadric hypersurface $Q$. Note that $\phi_{L^{2}}(C)$ is contained in $Q$, which is a cone over a surface $V$ isomorphic to $\mathbf{P}^{1} \times \mathbf{P}^{1}$, which has the point $p$ as it's vertex. Now by injectivity of $\bar{\mu}_{L}$, the composition of the morphism $\phi_{L^{2}}$ with the projection $\pi_{p}$ from the point $p$ coincides with the composition of $\phi_{L} \otimes \phi_{L}: C \rightarrow \mathbf{P}^{1} \times \mathbf{P}^{1}$ and the Segre embedding $\nu: \mathbf{P}^{1} \times \mathbf{P}^{1} \rightarrow \mathbf{P}^{3}$. Consider also that, $\pi_{p}\left(\phi_{L^{2}}\right)(C)$ is contained in $V$ whose generating lines cut on $C$ the pencil $L$. Let $\sigma: U \rightarrow V$ be the blow up of $V$ at the conductor ideal of $\pi_{p}\left(\phi_{L^{2}}\right)(C)$, and $C^{\prime} \subset U$ the proper transform of $\pi_{p}\left(\phi_{L^{2}}\right)(C)$. Then the adjoint morphism $\phi_{K_{U}+C^{\prime}}: U \rightarrow \mathbf{P}^{7}$ maps $U$ onto a surface containing $k(C)$ which contains a 1-parameter family of rational normal curves $L_{s}$ of degree 4 which cut the vertex $\Gamma$ in two points. Now, by the following lemma it follows that these curves are the curves $\Gamma_{s}$ and $F_{L}$ is birational with $\mathbf{P}^{1} \times \mathbf{P}^{1}$.

Lemma 10. Let $R$ and $R^{\prime}$ be rational curves in $\mathbf{P}^{4}$, which have 6 points in common and cut a line $L$ at least in two points. Then $R=R^{\prime}$. 
Proof. Let $L$ be the line which $R$ and $R^{\prime}$ cut it in two points. By projecting from $L$ to $\mathbf{P}^{2}$, we get two conics $\pi(R)$ and $\pi\left(R^{\prime}\right)$ which cut each other in 6 points. This is possible only when $\pi(R)=\pi\left(R^{\prime}\right)$. But this equality means that there are infinitely many planes $\Lambda$ which cut the rational curves $R, R^{\prime}$ simultaneously and contain the line $L$. Take one such a plane. The rational curves $R$ and $R^{\prime}$ have 6 points in common and cut the plane at least in three points. Now the lemma follows by lemma (4.3) of [2].

By a same method in [3], it is easy to see that the variety $F=\cup_{s \in S} \Gamma_{s}$ should be of dimension at least 3 , otherwise, for any line bundle $L^{\prime}$, we have $F_{L}=F_{L^{\prime}}$ and it follows that the canonical curve $k(C)$ has a $\mathbf{g}_{6}^{r}$, with $r \geq 2$, which is impossible by generality of curve. Now, the corollary 9 is valid. Indeed, for a general member $s$ of $S, k(C)=\overline{\cup_{s \in S} \Gamma_{s}}$ when $\operatorname{dim}\left(\Gamma_{s}\right)=0$. Then assume that $\operatorname{dim}\left(\Gamma_{s}\right)=1$. Notice that $\Psi_{s}$ does not vanishes identically since $\operatorname{dim} F \geq 3$. Therefore, for a variety $H$,

$$
\overline{\cup_{s \in S} H_{s}}=k(C) \cup H .
$$

This shows that $k(C)$ is a component of the closure of the union of those locus where $\Psi_{s}$ drops rank twice.

\section{Acknowledgment}

I am grateful to Prof. E. Sernesi, who taught and introduced me to this topic. I benefited from warmly and energetic discussions which I had with him, when I was an Erasmus visitor in Rome Tre university. I am also thankful for Prof. C. Ciliberto, and A. Bruno for having friendly and useful discussions, about this subject and many other problems, during the period.

\section{References}

[1] C. Ciliberto, E. Sernesi, Singularities of Theta Divisor and Congruences of Planes, Journal of Algebraic Geometry, 1, 231-250 (1992).

[2] C. Ciliberto, E. Sernesi, Singularities of Theta Divisor and Families of Secant Spaces to a Canonical Curve, J.Algebra, 171, 867-893 (1995)

[3] C. Ciliberto, E. Sernesi, On the Geometry of Canonical Curves of Odd Genus, Communications in Algebra, 28(12) (2000), 5993-6001.

[4] C. Ciliberto, E. Sernesi, Projective Geometry Related to the Singularities of Theta Divisors of Jacobians, preprint.

[5] D. Eisenbud, Linear Sections of Determinantal Varieties, Amer. J. Math., 110 (1988), 541575 . 
[6] Ph. Griffiths, J. Harris, On the Variety of Special Linear Systems on a General Algebraic Curve, Duke Mathematical Journal, v.47, No. 1(1980), 233-272

[7] J. Harris, Algebraic Geometry, A First Course, 1992, Springer Verlag.

[8] R. Hartshorne, Algebraic Geometry, Graduate Texts, 1974, Springer Verlag. 